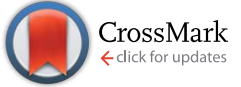

Cite this: RSC Adv., 2017, 7, 8060

Received 24th November 2016 Accepted 18th January 2017

DOI: 10.1039/c6ra27260f

www.rsc.org/advances

\section{Nanoparticle functionalized laser patterned substrate: an innovative route towards low cost biomimetic platforms $\uparrow$}

\author{
K. Bagga, ${ }^{\star a b}$ R. McCann, ${ }^{\text {abc }}$ F. O'Sullivan, ${ }^{d}$ P. Ghosh, ${ }^{e}$ S. Krishnamurthy, ${ }^{e}$ A. Stalcup, ${ }^{\text {bf }}$ \\ M. Vázquez ${ }^{\text {abf }}$ and D. Brabazon ${ }^{\text {abc }}$
}

Integration of nanotechnology and advanced manufacturing processes presents an attractive route to produce devices for adaptive biomedical device technologies. However, tailoring biological, physical, and chemical properties often leads to complex processing steps and therefore to high manufacturing cost impeding further scalability. Herein, a novel laser-based approach is introduced to manufacture low cost biocompatible polymer substrates functionalized with ultrapure nanoparticles. Laser direct writing was performed to create micron-sized patterns on $188 \mu \mathrm{m}$-thick cyclic olefin polymer (COP) substrates using a picosecond pulsed $1064 \mathrm{~nm}$ Nd:YAG laser. The Pulsed Laser Ablation in Liquids (PLAL) technique was exploited in this work to prepare colloidal solutions of ultrapure nanoparticles to impart bio-functionality onto laser patterned surfaces. Combining the laser patterns and their modification with PLAL-nanoparticles resulted in a functional and biocompatible substrate for biosensing applications. Our in vitro cell viability studies using a model cell line (human skin keratinocyte, $\mathrm{HaCaT}$ ) suggest that these nanoparticles immobilized on the surfaces function as a biomimetic platform with the ability to interact with different biological entities (e.g. DNA, antibodies etc.).

\section{Introduction}

To meet the global demand for low cost, disposable and adaptive biomedical technology, it is very important to achieve strategic convergence of different technologies such as nanotechnology, medicine and manufacturing processes. Over the

\footnotetext{
${ }^{a}$ Advanced Processing Technology Research Centre, School of Mechanical Engineering, Dublin City University, Glasnevin, Dublin 9, Ireland. E-mail: komal.bagga@dcu.ie; Tel: $+353-1-700-7603$

${ }^{b}$ Irish Separation Science Cluster, National Centre for Sensor Research, Dublin City University, Glasnevin, Dublin 9, Ireland

${ }^{c}$ National Centre for Plasma Science and Technology, Dublin City University, Glasnevin, Dublin 9, Ireland

${ }^{d}$ National Institute of Cellular Biology, Dublin City University, Glasnevin, Dublin 9, Ireland

${ }^{e}$ Materials Engineering, The Open University, Milton Keynes, MK7 6AA, UK ${ }^{f}$ School of Chemical Sciences, Dublin City University, Glasnevin, Dublin 9, Ireland $\dagger$ Electronic supplementary information (ESI) available: XPS spectra of laser treated sample (Fig. 1S), Fig. 2S: scanning electron microscopy images of the COP substrate at test parameters, NMR spectroscopy spectra of laser textured COP (Fig. 3S-5S), water contact angle measurements for 5 day measurement period after different plasma treatments (Fig. 6S). SEM image of carbon nanoparticles deposited on non-textured COP (Fig. 7S). Fluorescent microscopy images of nuclei stained HaCaT cells on non-textured non NP functionalized substrate (Fig. 8S). Fluorescent microscopy images of nuclei stained HaCat cells after exposure of SiOx NPs and TiOx NPs (Fig. 9S). Table 1S: laser conditions for different patterns, Table $2 \mathrm{~S}$ : cell count on each substrate post 7 day exposure to different concentration of CNps. See DOI: 10.1039/c6ra27260f
}

last few years, there have been some significant advances in the development of new and innovative products in an attempt to realize this demand. Most of the effort is in the field of developing biosensors which constitute a US $\$ 12.9$ billion market and is projected to expand to US $\$ 21.17$ billion by 2023. ${ }^{1}$ For example, biosensors are particularly applicable in testing blood glucose levels, HIV, and early cancer detection, which are areas of great medical relevance. ${ }^{2}$ The upsurge in applications of biosensors is mainly driven by the need for advanced diagnostic solutions for the rising number of prevalent diseases requiring ultrasensitive detection. Biosensors, in simple terminology, can be defined as substrates (plastic, polymer, silicon or fused quartz) onto which 'capture' probes are attached to detect the 'target' (e.g., biological pathogens, gene sequences, illegal drugs, chemical toxins or environmental pollutants). They present a powerful molecular technology allowing selective and sensitive detection of small changes in target molecule concentrations for accurate diagnosis. A general on-going aim is to achieve this also within shorter sample processing times. However, to achieve these goals a comprehensive chemical and physical analysis is required in finalizing the biosensor design, including optimal selection of substrate, surface functionalization and detection system.

Biosensor substrates should ideally be inexpensive, mass producible, easily adaptable to detecting other biological agents, robust, and simple to use. Additionally, for some applications, it is crucial to create a biocompatible environment for further 
functionalization, such as attaching bioactive molecules. ${ }^{3}$ Keeping in mind these prerequisites, we have established an innovative laser based approach to manufacture low cost, stable and functionalized biocompatible polymer substrates.

Polydimethylsiloxane (PDMS), poly(methylmethacrylate) (PMMA), polycarbonate (PC), polyester (PES) and polystyrene (PS) are the most common polymers used to fabricate biomedical devices. However, to address the ongoing challenges of cost, suitable physical, chemical and biological properties, biomaterial scientists have become adaptive in their choice of substrate materials. Recently, cyclic olefin polymers (COPs), have emerged as a promising substrate for biomedical devices and lab-on-achip applications, ${ }^{4,5}$ because of their exceptional properties, such as mechanical strength, high chemical resistance, optical transparency and low auto fluorescence thereby allowing efficient optical detection. Additionally, this class of polymers are compliant with USP class VI and/or ISO 10993 standards, "Biological Evaluation of Medical Devices". ${ }^{6}$

The next step after choosing a suitable substrate is the device fabrication. Techniques such as photolithography, electron beam lithography and reactive ion etching offer the ability to imprint micro/nanoscale features, allowing reduction in the size of the analytical system as well as analyte detection time period. While these techniques permit a degree of control over morphology, they involve a series of processes (defining pattern, material deposition, plasma cleaning, lift off and etching) which render them less viable for device commercialization. In this work, we have utilized direct laser writing technology on COP for device manufacturing. To the best of our knowledge, very few studies have examined the feasibility of using industrial $1064 \mathrm{~nm}$ Nd:YAG lasers for the processing of $\mathrm{COP}^{7,8}$ Some important advantages of this technology are that it is fast, reproducible, clean, cost effective and produces a readily modifiable surface through micro- and nanoscale structures in one step. ${ }^{9}$

After the substrate processing, the next challenging issues are to impart biofunctionality to the device substrate ${ }^{10}$ and enhance device sensitivity. In this context, nanomaterials have successfully replaced traditional dyes/fluorophores because of their exquisite material and optical properties imparting sensitivity in chemical and biological sensing. ${ }^{11}$ Advances in nanomaterial synthesis have produced a plethora of chemical and physical techniques that can be employed to produce nanoparticles (NPs). Of these, the Pulsed Laser Ablation in Liquids (PLAL) technique is gaining a lot of interest because the nanomaterials produced are surfactant-free with "bare" nanomaterial surfaces. ${ }^{12,13}$ During PLAL, an intense laser beam irradiates the surface of a solid target resulting in plasma plume generation at the liquid-solid interface, with ejection/formation of nanoclusters of the constituent material in the confining medium, thereby leading to formation of ultrapure colloidal nanoparticles in solution. ${ }^{14}$ The resulting bare surface of the NPs is highly desirable for further functionalization as it favors access to the NP's surface active sites. Additionally, the absence of ligand or stabilizer molecules on the surface of PLAL-NPs, promotes higher chemical activity in comparison to conventionally synthesized NPs. ${ }^{15}$ In this work, we have synthesized ligand-free carbon nanoparticles (CNps) via PLAL and applied them as the functional material on our laser patterned substrates. CNps were chosen because of their superior biological properties such as low toxicity, biocompatibility and their successful demonstration in bioimaging, biosensor and biomolecular/drug delivery applications..$^{16,17}$

To adopt these substrates for bio-applications, it is imperative to study their biocompatibility using in vitro tests with a model cell line. Creating bio-functional substrates is crucial for biosensing applications, as apart from providing the physical support, the functional groups or nanoparticles immobilized on the surface function as a biomimetic platform with the ability to interact with different biological entities (e.g. DNA, antibodies etc.). Numerous successful biocompatibility studies have been performed on pristine COP film, ${ }^{18-20}$ and the result opens up the possibility of using COP as bio-platform in sensing and diagnostic applications. However, there has been no studies on CNp functionalized laser patterned COP substrates. Hence, to establish the use of our novel substrates for biological applications, we performed cell viability studies using human skin keratinocyte cell line, HaCaT, derived from immortalized human keratinocytes. This cell line is considered an appropriate model for studying the factors that might regulate keratinocytes growth and toxicity, ${ }^{21}$ as it showcases morphological and functional properties of normal epidermal keratinocytes including proliferation and differentiation. ${ }^{22}$

To summarize, $1064 \mathrm{~nm}$ Nd:YAG laser was employed under ambient conditions to fabricate different patterns on COP with varied features (e.g., size, shape, morphology, etc.) by tuning laser parameters (e.g., laser fluence, number of passes). Scanning electron microscopy and 3D optical profilometry was performed to examine the surface morphology and for dimensional analysis of the microchannels. X-ray photoelectron spectroscopy (XPS) and Nuclear Magnetic Resonance (NMR) spectroscopy were performed to examine the nature of chemical bonding and the interactions of laser processing on the surface chemistry of COP. To overcome the inherent hydrophobicity of COP surfaces, a systematic investigation on the effect of different plasma treatments was carried out on surface of COP. Subsequently, the laser textured substrate was functionalized with ultrapure CNps via PLAL technique and were characterized by spectroscopy techniques (UV-Vis, photoluminescence) and Transmission Electron Microscopy. Finally, to confirm their applicability as low cost biomimetic platform for adaptive biomedical technology, in vitro cell viability studies using a model cell line (human skin keratinocyte, HaCaT) were performed.

\section{Materials and methods}

\subsection{Materials}

ZeonorFilm ZF14-188 (Zeon) cyclic olefin polymer $(188 \mu \mathrm{m}$ thick) purchased from Ibidi, Germany, was used as the device substrate material. Prior to laser processing the polymer sheet was cut $(2 \times 2 \mathrm{~cm})$ and cleaned with isopropanol (Sigma Aldrich) in an ultra-sonication bath, rinsed with deionized water (Milli-Q water purification system) and dried using compressed clean dry air to ensure that the film was pristine and free from any surface impurities. For nanoparticle generation, a graphite target with $99.999 \%$ purity was obtained from 
Sigma Aldrich in cylindrical form with a diameter of $6 \mathrm{~mm}$ and cut to a height of $8 \mathrm{~mm}$ with a diamond blade saw.

\subsection{Substrate patterning}

A 1064 nm Q-switched, diode-pumped solid-state neodymiumdoped yttrium aluminum garnet laser (Nd:YAG) from BrightSolutions 1064 WEDGE HF (Fig. 1A) was employed for the direct laser process.

A 2D scanning galvanometer (Raylase SS-12) was used to raster the beam in the $x y$-plane, and a movable z-stage (PI M-404 $4 \mathrm{PD}$ ) was used to control the position of the sample. The laser beam was focused to a spot size of $140 \mu \mathrm{m}$ and scanned unidirectionally across the sample at a speed of $1.2 \mathrm{~mm} \mathrm{~s}^{-1}$ to form parallel channels separated by $200 \mu \mathrm{m}$. The COP films were mechanically fastened to the sample stage for the duration of processing. Further details of the processing parameters are listed in Fig. 1B.

\subsection{Nanoparticle synthesis}

Laser ablation experiments were carried out using the same laser as for direct laser writing experiments on COP substrates. For the generation of carbon nanoparticles, the graphite target (99.999\% pure from Sigma Aldrich) in cylindrical form with a diameter of $6 \mathrm{~mm}$ and height of $8 \mathrm{~mm}$ was placed on the bottom of a glass cuvette (dimensions $10 \times 10 \times 50 \mathrm{~mm}$ ). The cuvette was filled with $2 \mathrm{~mL}$ deionized water (DI water) which corresponded to $1 \mathrm{~cm}$ of liquid above the flat circular surface of the target.

The laser beam (repetition rate: $10 \mathrm{kHz}$ ) was focused onto the target material using a lens with a focal length of $30 \mathrm{~cm}$. During all of the experiments, the graphite target was held in the focal plane of the laser beam. A 2-dimensional scanning galvanometer was used to scan the laser beam across the top of the graphite target in a circular pattern, at a scan rate of $1.2 \mathrm{~mm} \mathrm{~s}^{-1}$. The laser fluence was measured experimentally using a 30A-P-17 OPHIRÒ power meter. For the production of CNps, the ablation was carried out at $0.71 \mathrm{~J} \mathrm{~cm}^{-2}$ fluence for a $30 \mathrm{~min}$ irradiation time. The formation of carbon nanostructures could be visualized by the slight change of the color (from transparent to varying tones of grey) of the liquid during ablation. However, it should be noted that changes in the color of the colloidal solution were only recorded for high fluence after a few minutes of ablation. The characterization measurements were performed one day after preparation of the colloids.

\subsection{Sample preparation}

Two-step sample preparation was used in which plasma treatments of laser textured COP is conducted first and the nanoparticle deposition is done afterwards.

To increase hydrophilicity and facilitate the NP deposition on substrates, a systematic investigation was carried out to study the effect of the different plasma treatments on the surface wettability of the substrates. Four different plasma treatments were conducted to tune the hydrophilicity of the substrate, (i) 5 minute $\mathrm{Ar} / \mathrm{O}_{2}$ reactive ion etch (RIE) (Oxford Instruments PlasmaPro $80 \mathrm{RIE}$ tool), (ii) 5 minute $\mathrm{O}_{2}$ plasma clean, (iii) 10 minute $\mathrm{O}_{2}$ plasma clean, (iv) piranha (sulphuric acid/hydrogen peroxide at $4: 1$ ) etch clean followed by 5 minute $\mathrm{O}_{2}$ plasma clean. Treatment (ii) to (iv) were conducted using a Harrick PDC-32G plasma cleaner.

Following the plasma treatment, the colloid was deposited onto the laser patterned substrates to functionalize the textured pattern. Drop-casting, a straightforward and easy deposition method, was employed. Nanoparticle dispersion was spread onto a substrate, and left for evaporation under controlled conditions $\left(37^{\circ} \mathrm{C}\right)$ in an incubator from Mason Technology. The film thickness could be tuned with the volume and concentration of NP dispersion.

\subsection{Characterization}

2.5.1. Substrate characterization. Surface topographies were examined by Scanning Electron Microscopy using an EVO LS15 (Zeiss) with $\mathrm{a} \mathrm{LaB}_{6}$ filament, accelerating voltage of $10 \mathrm{kV}$, and a beam current intensity of 25 pA. Samples were gold coated using a ScanCoat Six (Edwards) sputter coater set at a deposition current of $25 \mathrm{~mA}$ for $80 \mathrm{~s}$, resulting in a coating thickness of $34 \mathrm{~nm}$. The shape of the micro-channels was examined by imaging the cross-sectional SEM.

The contact angles $(\theta)$ of laser treated olefin polymer surfaces were measured with a sessile drop goniometer (FTA 200 angstrom, USA) using DI water in atmospheric conditions.

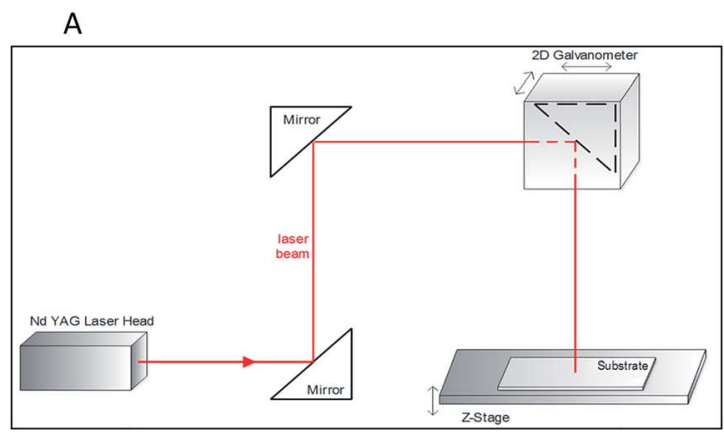

\begin{tabular}{|ccc}
\hline Brocess parameter & Single Pass & Multiple pass \\
\hline Fluence & $0.15-0.78 \mathrm{~J} / \mathrm{cm}^{2}$ & $0.51 \mathrm{~J} / \mathrm{cm}^{2}$ \\
\hline Scan Rate $(\mathrm{mm} / \mathrm{s})$ & 1.2 & 1.2 \\
\hline Beam diameter $(\mu \mathrm{m})$ & 140 & 140 \\
\hline Pulse Width $(\mathrm{ps})$ & 750 & 750 \\
$\begin{array}{c}\text { Pulse Repetition } \\
\text { Frequency }(\mathrm{kHz})\end{array}$ & 7.5 & 7.5 \\
No. of passes & 1 & $2-10$ \\
\hline
\end{tabular}

Fig. 1 (A) Schematic of the $1064 \mathrm{~nm}$ Nd:YAG laser processing system used for the experiment. (B) Process parameters used during laser processing of COP substrates. 
The profile of the microchannels was examined using a VHX2000 (Keyence) 3D Optical Microscope. A $68 \mathrm{~nm}$-thick film of gold was coated onto the sample substrates using the ScanCoat Six sputter, to reduce the transmission in the visible range by approximately $90 \%{ }^{23}$ and allow the sample surface to be imaged optically. Images were taken at steps of $0.43 \mu \mathrm{m}$ from the base of the channel upwards at $1000 \times$ magnification with the error bars calculated for a $90 \%$ confidence interval.

X-ray photoelectron spectroscopy (XPS) measurements were carried out on a load-locked Kratos XSAM 800 apparatus equipped with a dual anode X-ray source using a nonmonochromatic AlK $\alpha$ radiation (photon energy $1486.6 \mathrm{eV}$ ) and a $150 \mathrm{~mm}$ hemispherical analyzer giving a total instrumental resolution of 0.85 . All spectra were calibrated using the $\mathrm{C} 1 \mathrm{~s}$ photoelectron component peak of amorphous carbon $(284.8 \mathrm{eV})$ present in the sample and data analysis was performed with Casa XPS software. The sample area analyzed was around $1 \times$ $1 \mathrm{~cm}$ with $4-8 \mathrm{~nm}$ the maximum analysis depth. The resolution of binding energy is estimated to be within $\pm 0.2 \mathrm{eV}$. Wide-scan XPS spectra show no impurities were induced in the samples during preparation or measurement (Fig. 1S in ESI $\dagger$ ). It should be noted that the sample was not prepared under ultra-high vacuum conditions, hence the fitting of spectrum for $\mathrm{COP}_{-}$ pristine sample could not be achieved respect to the theoretical one.

2.5.2. Nanoparticle characterization. Optical absorption spectra were recorded in a quartz cuvette $(10 \mathrm{~mm}$ path length, Helma) with a Varian Cary 50 UV-Vis spectrophotometer. The optical spectra were scanned within the range $200-1200 \mathrm{~nm}$ at $600 \mathrm{~nm} \mathrm{~min}{ }^{-1}$ scan rate with correction for water absorption. Photoluminescence of the colloidal solution was measured using a Jasco FP-8500 fluorescence spectrometer.

Transmission Electron Microscopy (TEM) was performed with a FEI Titan instrument, operating at $300 \mathrm{kV}$, equipped with a Field Emission Gun (FEG), spherical aberration corrector system (Cs-corrector) of the objective lens. The samples were prepared by drop-casting $40 \mu \mathrm{L}$ of the colloidal solution onto carbon coated 300 mesh copper grid and left to evaporate at room temperature.

\subsection{Biological experiments}

2.6.1. Cell culture. The human skin keratinocyte cell line, HaCaT, was obtained from (CLS) (Cell Lines Service D5671) supplemented with $2 \mathrm{mM}$ L-glutamine (Thermo Fisher Scientific, Cat no. 25030081) and 10\% v/v fetal calf serum. The cell line was cultivated in a $37{ }^{\circ} \mathrm{C}$ humidified incubator with $5 \%$ $\mathrm{CO}_{2}$. Test surfaces (patterned COP with and without NPs) were left under a UV light in a laminar flow overnight to sterilize. $\mathrm{GmbH}$. The HaCaT cell line was maintained in DMEM $(4500 \mathrm{mg}$ $\mathrm{L}^{-1}$ glucose) (Sigma Aldrich). The test surfaces were then placed into non-tissue cultured treated $35 \mathrm{~mm}$ dishes. The test surfaces were seeded with $2 \times 10^{5}$ cells per dish and incubated for 7 days prior to analysis and media were changed every second day.

2.6.2. Live/dead assay. The LIVE/DEAD Viability/ Cytotoxicity Assay Kit (Cat no. L3224) from Thermo Fisher Scientific was used to assess the growth and viability of the
HaCaT cells on the test surfaces. The protocol was followed in accordance to manufacturer's instructions. Briefly, wells were washed with PBS. The cells were incubated in serum free media with $5 \mu \mathrm{M}$ EthD-1 and $5 \mu \mathrm{M}$ calcein AM for $30 \mathrm{~min}$ at $37{ }^{\circ} \mathrm{C}$. Following incubation, the test surfaces were washed with PBS and placed inverted in fresh serum free media in CELLview 35 $\mathrm{mm}$ dishes with coverslip bottom (Greiner Bio-One, Cat no. 627861). Test Surfaces were imaged on a Nikon Ti-E with a Hammatsu ImagEM.

2.6.3. Imaging of live cell nuclei on test surfaces. The nuclei of HaCaT cells on the various test surfaces was assessed using NucBlue Live ReadyProbes Reagent (a formulation of Hoechst 33342) from Thermo Fisher Scientific (Cat no. R37605). Briefly, 2 drops of reagent was added to the cell culture and incubated at $37^{\circ} \mathrm{C}$ in $5 \% \mathrm{CO}_{2}$ for 20 minutes. Following incubation, the test surfaces placed inverted in fresh media in CELLview $35 \mathrm{~mm}$ dishes with coverslip bottom (Greiner BioOne, Cat No. 627861). Test surfaces were imaged using a Nikon Ti-E with a Hamamatsu ImagEM.

\section{Results and discussion}

\subsection{Substrate processing and characterization}

Direct laser writing is a very straight-forward technique, allowing for a variety of reproducible 2D patterns on cyclic olefin polymers (COP) films to be created through optimization of the laser processing parameters. Various patterns are demonstrated in Fig. 2a to d, including cross hatch pattern (Fig. 2a), a spiral pattern (Fig. 2b), text pattern (Fig. 2c) and light texture pattern (Fig. 2d). These were obtained with direct laser writing using the noted $1064 \mathrm{~nm}$ Nd:YAG laser. Top and bottom right images represents the 3D profile of patterns obtained for (a), (b), (c) and (d) respectively. The description of laser parameters utilized for each of the pattern are listed in the Table 1S ESI. $\dagger$

Our group was the first to report the fabrication of patterned structures on optically transparent $188 \mu \mathrm{m}$ thin COP film via a simple laser assisted technique in ambient atmosphere without the need of controlled gas chambers. ${ }^{24}$ This technique offers a low cost, innovative and flexible route to fabricate different patterns with varied features (size, shape, morphology, etc.) by tuning laser parameters (such as laser fluence and number of passes). Under optimized conditions, we have developed the fabrication process along with a software capable of converting the test patterns into the format readable by the laser direct writing system. To realize the application of these generated substrates in a lab-on-a-chip/biosensor format, several test experiments (not reported here) were performed to optimize the generated pattern. ${ }^{24}$ The principal criteria taken into consideration were the wettability, roughness, reproducibility and ease of functionalization. With these in mind, the fabrication routine was finalized. Fig. 3 shows the optical micrograph of the final patterned substrate obtained after laser processing. The SEM images of the microchannels are presented in Fig. 3: top view (Fig. 3b), a tilt of $10^{\circ}$ (Fig. 3c), and cross section view of a single channel (Fig. 3d). These channels were $70 \mu \mathrm{m}$ wide, $60 \mu \mathrm{m}$ deep, clean and uniform microchannels, with a $200 \mu \mathrm{m}$ interchannel distance. 


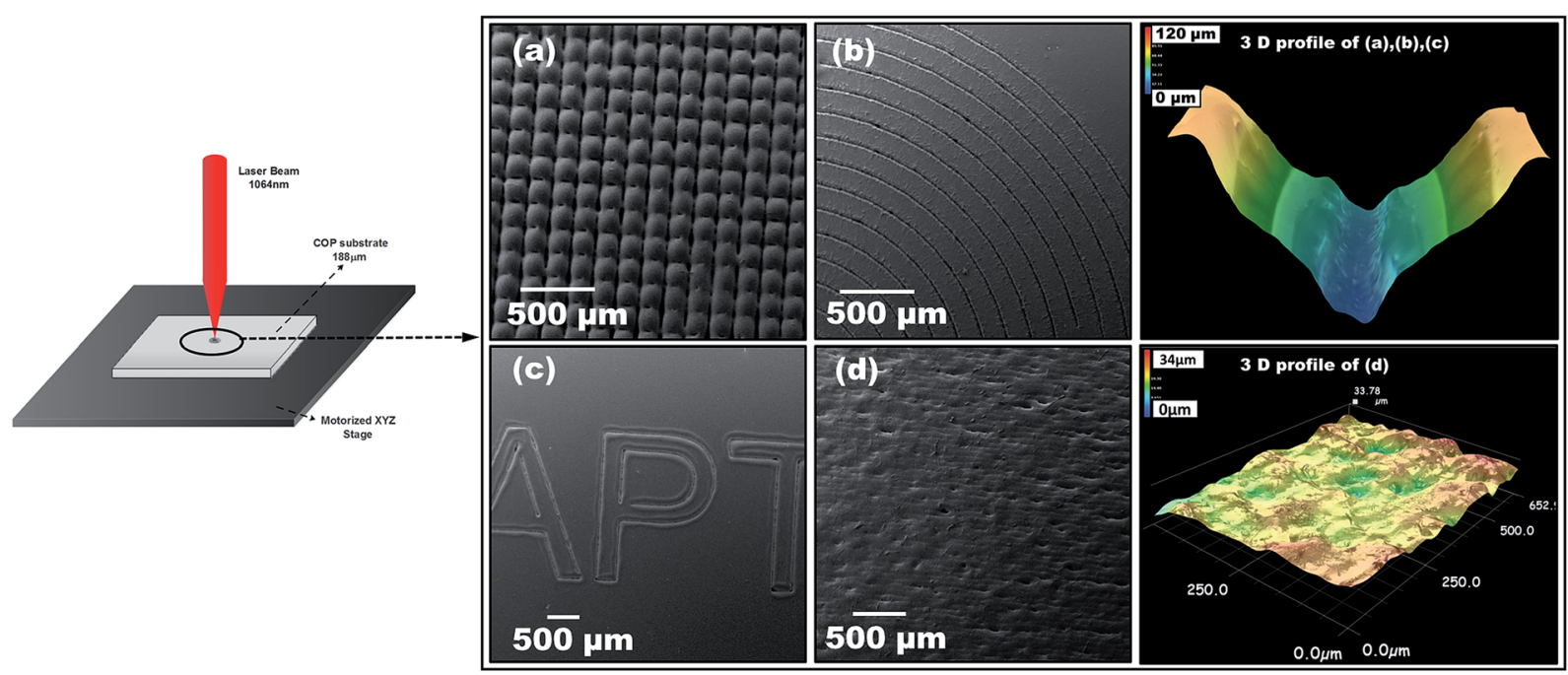

Fig. 2 (Left) Schematic of the laser direct writing (LDW) process to fabricate patterns under ambient conditions; (right) top view SEM images of different patterns obtained with direct laser writing process (a) cross hatch pattern, (b) concentric circles, (c) text pattern, and (d) light texture pattern. Top right: 3D profile of patterns obtained for $(a-c)$. Bottom right: 3D profile of pattern obtained from (d).

Measurements using 3D profilometry (Fig. 3e inset) revealed a V-shaped profile of microchannels, consistent with theoretical predictions..$^{25}$ It should be noted that in some cases, due to light ray blockage the $3 \mathrm{D}$ profile measurement of the channels compared to SEM measurement, does not give highly precise depth of channels. Some re-cast ablation melt can be seen on the crests of the channels, however, this can be minimized by carrying out the laser processing within the range of fluence examined.

With optimum thermal diffusion at the ablation site, localized melting of condensed debris can be induced. Based on the control experiments (refer Fig. $2 \mathrm{~S}$ in ESI†), the optimal laser conditions for laser processing of COP was a fluence of $0.63 \mathrm{~J}$ $\mathrm{cm}^{-2}$ and a pulse width of $700 \mathrm{ps}$. These conditions were above the ablation threshold (of approximately $0.32 \mathrm{~J} \mathrm{~cm}^{-2}$ ) but not sufficient for any laser induced damage to occur.

From a manufacturing standpoint, it is desirable to have micro/nano-scale features that can be scaled up to large areas at low cost and high throughput. Under the optimized condition, our low-cost patterning technique can be readily applied for large-scale uniform pattern production. In that regard, we have identified and tested the laser set-up parameters that can be incorporated into our current laser experimental setup to produce uniform patterning geometries over large areas. One of the key parameters was to introduce a cold plate under the motorized stage to ensure uniform thermal environment and minimize the heat buildup in the processed area at higher fluence and at longer processing times. Utilization of the cold plate reduced the localized damage which otherwise lead to substrate warping and the appearance of large bubble-like formation $\mathrm{s}^{24}$

To investigate the effect of laser ablation on surface chemistry of the COP, X-ray photoelectron spectroscopy (XPS) analysis was performed. Wide-scan XPS spectra confirmed the absence of any impurities induced in the samples during preparation or measurement (Fig. 1S ESI $\dagger$ ).

Fig. 4 represents the high resolution scan for the core level photoemission spectra of $\mathrm{C} 1 \mathrm{~s}$ spectra obtained on (a) pristine

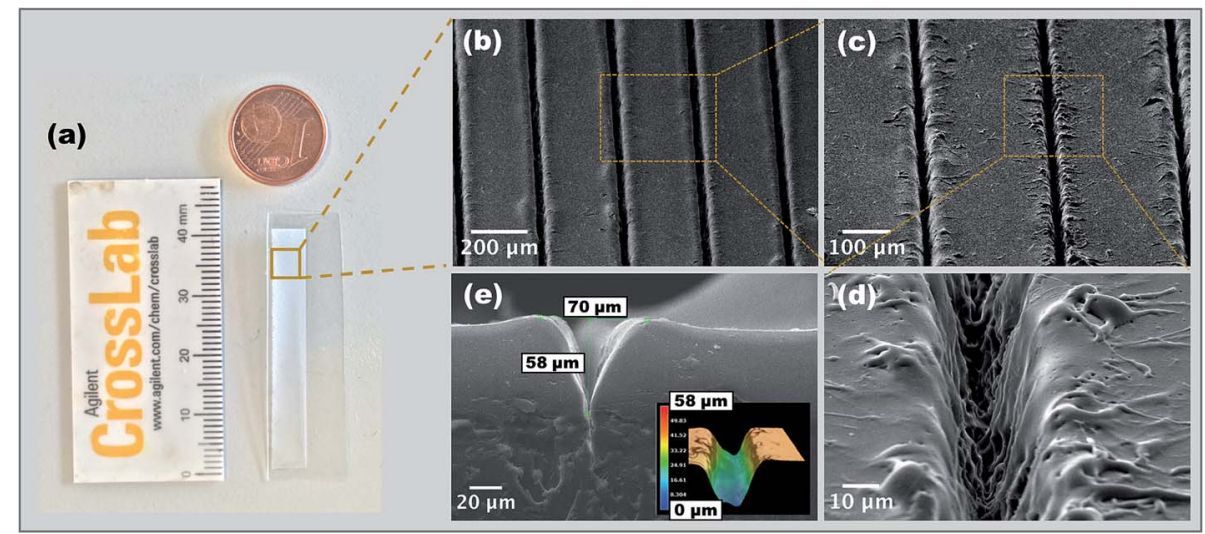

Fig. 3 (a) Picture of device substrate showing patterned array obtained by the $1064 \mathrm{~nm}$ laser direct writing technique. SEM images of the microchannels at different magnifications $(b-d)$ and cross-section (e). 

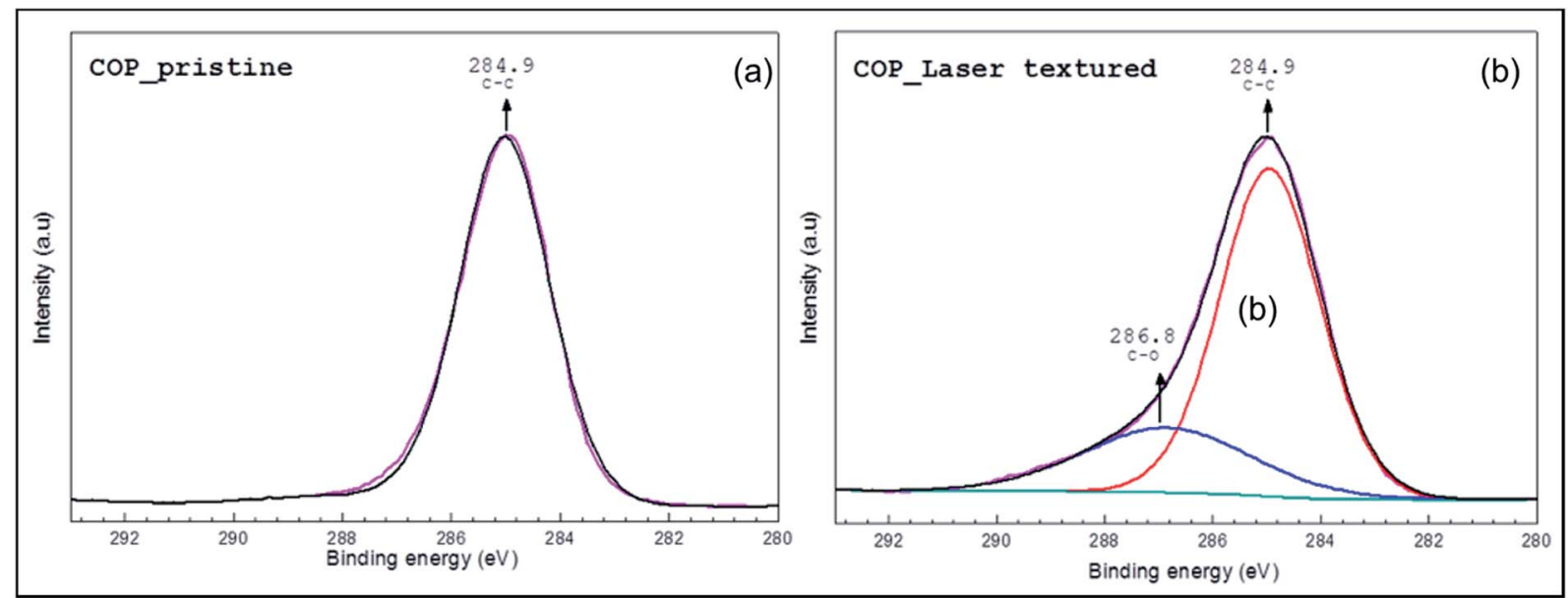

Fig. 4 X-ray photoelectron spectroscopy (XPS) spectra of (a) pristine COP surface and (b) laser textured COP surface at a fluence of $0.63 \mathrm{~J} \mathrm{~cm}^{-2}$.

and (b) laser textured COP substrate. Data analysis and curve fitting were performed using CasaXPS software. The C 1s carbon component and corresponding peak were all fitted with Lorentzian peaks convoluted with the same Gaussian employed for broadening the $\mathrm{sp}^{2}$ related peak. The Gaussian component accounts for the instrumental energy resolution together with any chemical disorder, and the Lorentzian width accounts for the finite core hole lifetime associated with the photoionization process. The background photoelectron intensity was subtracted by the Shirley method.

As expected, a saturated hydrocarbon peak at $\sim 285.0 \mathrm{eV}$ characteristic of $\mathrm{C}-\mathrm{C}$ from the COP polymer was recorded for both the pristine and laser treated samples. This is as a result of adventitious carbon generally comprised of polymeric short chains. ${ }^{26}$ However, the asymmetric $\mathrm{C}$ 1s spectrum could be fitted with two main peak components with binding energies of about, $285.0 \pm 0.2 \mathrm{eV}$ and $286.3 \pm 0.2 \mathrm{eV}$, attributable to the C-
$\mathrm{C} / \mathrm{C}-\mathrm{H}$ and $\mathrm{C}-\mathrm{O}$ species, respectively. This may be as a result of surface thermal oxidation, similar to that reported in literature ${ }^{27}$ which in our case is induced during the laser processing.

To eliminate the possibility of other functional groups, and confirm the presence of $\mathrm{C}-\mathrm{O}$ bond, we performed NMR analysis. The obtained ${ }^{1} \mathrm{H}-\mathrm{NMR}$, Correlation Spectroscopy (COSY) NMR and Heteronuclear Multiple Quantum Coherence (HMQC) NMR spectra confirmed the presence of the $\mathrm{C}-\mathrm{O}-\mathrm{H}$ hydroxyl moiety in the sample (Fig. 3S, 4S, $5 \mathrm{~S}$ respectively in the ESI $\dagger$ ).

\subsection{Surface modification}

Plasma treatment is widely used to impart hydrophilic characteristics onto surfaces to facilitate and promote further surface functionalization. Pristine COP film has a water contact angle (WCA) of $88^{\circ}$, is effectively self-passivated and under atmospheric exposure undergoes minimal oxidation. Systematic
A

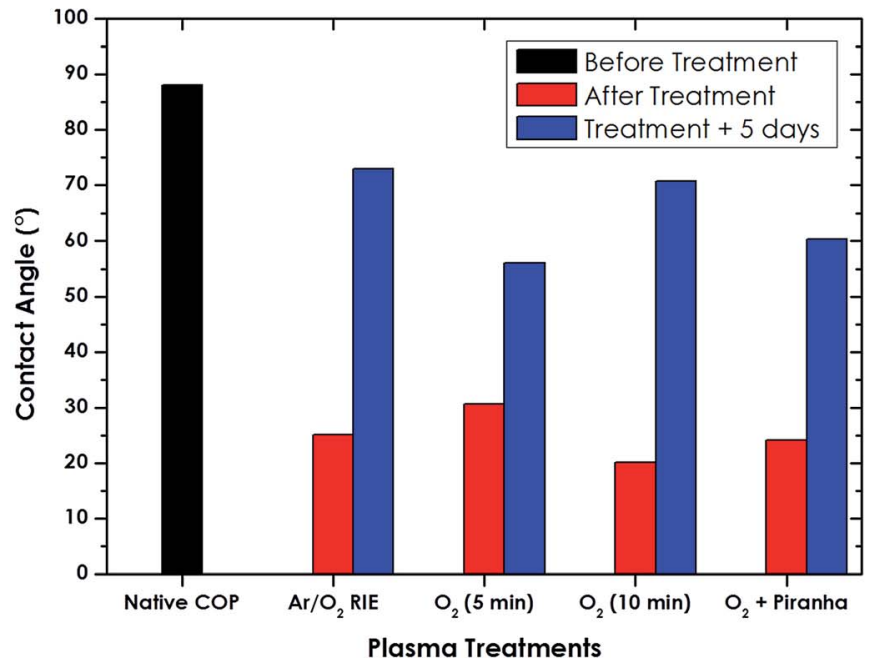

B
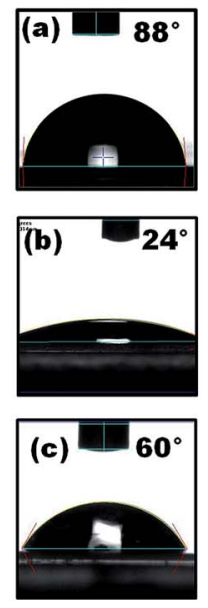

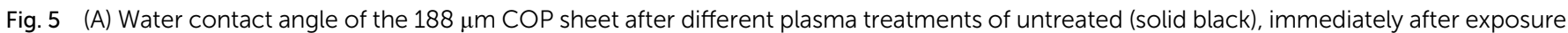

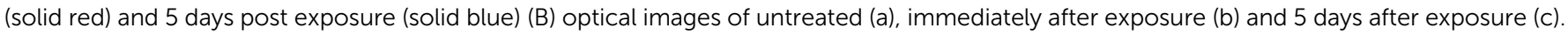


investigation was carried out to study the effect of the different plasma treatment on surface wettability by conducting WCA measurements. Additionally, WCA measurements were performed after 5 day post-plasma treatment to study the stability of the induced hydrophilicity and its recovery over time. Fig. 5 shows the initial and 5 day post-treatment water contact angle of the $188 \mu \mathrm{m}$ COP sheet after different plasma treatments.

It is clearly observed that all the treatments result in rapid decrease of the water contact angle from $88^{\circ}$ to $\sim 20-30^{\circ}$ but this increased progressively over the course of the 5 day measurement period (Fig. $6 \mathrm{~S}$ in the ESI $\dagger$ ). In case of $\mathrm{Ar} / \mathrm{O}_{2}$ RIE treatment, WCA return to the highest value $\left(\sim 73^{\circ}\right)$, while the 5 minute plasma clean, both with and without the piranha (sulphuric acid/hydrogen peroxide at $4: 1$ ) etch treatment, stabilized at $60^{\circ}$. The 10 minute $\mathrm{O}_{2}$ plasma clean had the lowest treated contact angle but recovered after five days to a value $\left(\sim 70^{\circ}\right)$, close to that of the $\mathrm{Ar} / \mathrm{O}_{2} \mathrm{RIE}$ sample.

Characterizing the duration of the plasma-induced hydrophilicity is critical for mapping out a stable working window to perform further surface functionalization. Traditional polymers like polydimethylsiloxane (PDMS) have been reported to recover its wettability characteristics readily over time, ${ }^{28}$ thereby making them less desirable for applications requiring multiple functionalization. These results indicate the possibility of controlling and retaining the surface hydrophilicity of COP substrates for extended measurement periods when performing surface modification.

\subsection{Nanoparticle synthesis and characterization}

Carbon nanoparticles (CNps) were prepared in DI water by ablating high purity grade (>99.9995\%) graphite with $1064 \mathrm{~nm}$ laser pulse (Fig. 6a). Optical properties of the ligand-free CNps synthesized in DI water after $30 \mathrm{~min}$ of irradiation were investigated (see Fig. 6b).

A broad continuous band between $200 \mathrm{~nm}$ and $500 \mathrm{~nm}$ is observed (blue line) with a distinctive shoulder at around $260 \mathrm{~nm}$. The observation of characteristic absorption peak at wavelength of $260 \mathrm{~nm}$ and strong background absorption at wavelength up to about $500 \mathrm{~nm}$ establishes the successful generation of CNps with average size less than $50 \mathrm{~nm} \cdot{ }^{29,30}$ Moreover, the CNp colloid was excited at $360 \mathrm{~nm}$ to record photoluminescence spectra (Fig. 6b). A strong blue-green photoluminescence centered at around $430 \mathrm{~nm}$ was observed which is in accordance with previously reported works which shows luminescence from CNps due to radiative recombination of excitons at the particle surface during plasma assisted generation. ${ }^{31}$

Transmission electron microscopy was performed to obtain detailed information about the morphology and size distribution of the CNps in DI-water. TEM images in Fig. 7a shows an overall size distribution of CNps colloid (2-100 nm) obtained by PLAL with corresponding size histogram, Fig. $7 \mathrm{~b}$ represents NPs of size $10-50 \mathrm{~nm}$. NP of size around $2-5 \mathrm{~nm}$ are shown in Fig. $7 \mathrm{c}$ and d gives insight in crystallinity of single NP.

Non-agglomerated and isolated CNps with a spherical morphology were observed. The TEM analysis (Fig. 7a) reveals size distribution of CNps from 2 to $100 \mathrm{~nm}$ with a mean size of around $30 \mathrm{~nm}$ were produced via PLAL of graphite in DI water. Given the peculiarity of sample preparation of TEM grid (i.e. drop-casting colloidal NP solution onto carbon grid), nonlocalized distribution of NP sizes can be observed. For example, uniformly distributed NP of size 10-50 nm and $\sim 2.5 \mathrm{~nm}$ can be seen in Fig. 7b and c respectively. Well-resolved lattice fringes are observed in high-resolution TEM images, corresponding to interplanar spacing values of $0.32 \mathrm{~nm}$ (Fig. $7 \mathrm{~d}$ inset), which are close to (002) diffraction planes of graphite carbon $^{32}$ indicating the graphitic nature of the CNps.

The obtained nanoparticle solution was then drop-cast onto plasma modified laser textured COP and examined via SEM (Fig. 7S in the ESI $\dagger$ ). Further biocompatibility studies were performed on the NP functionalized substrate (discussed in Section 3.4.) and its potential use as model biomimetic platform for applications from sensing, to wearable or implantable devices, and to fundamental studies of cell growth and interactions.

\subsection{Biological studies}

To realize the application of any substrate as a possible biomimetic platform, it is very important that the developed platform aids in cell proliferation, growth and differentiation.

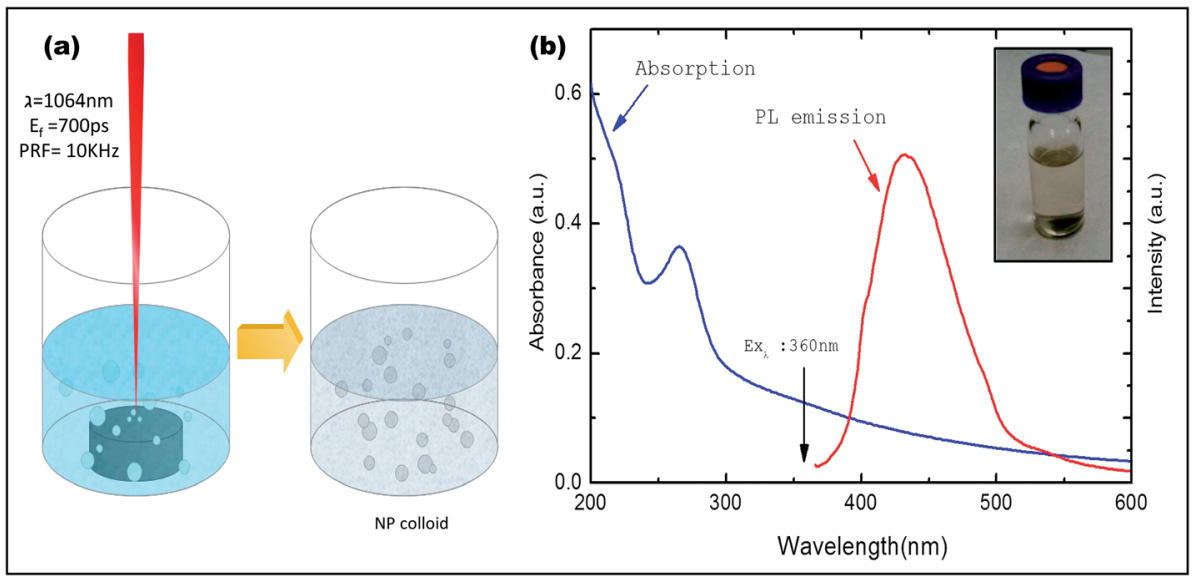

Fig. 6 (a) A schematic diagram of the experimental setup of laser ablation of graphite target in DI water (b) optical properties of the CNps colloidal solution absorption spectrum (blue line) and PL spectrum (red line). Inset: optical image of obtained CNps colloidal solution via PLAL. 

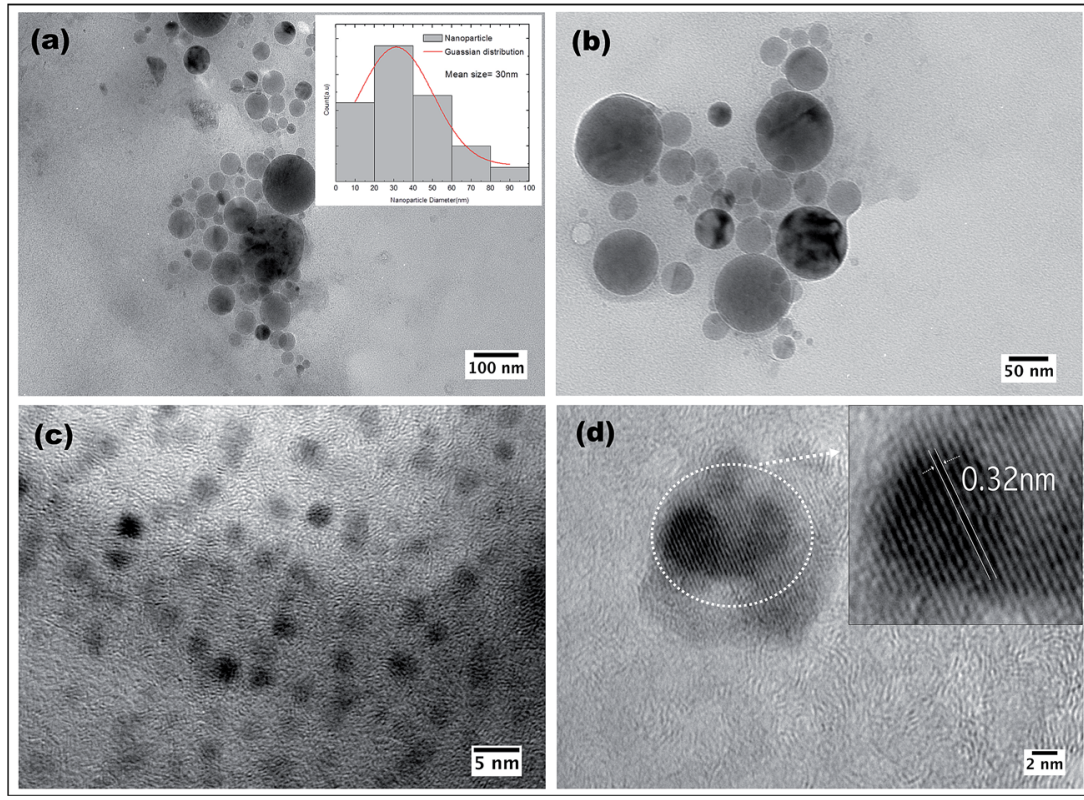

Fig. 7 (a)-(c) TEM images of CNps synthesized in water via $1064 \mathrm{~nm}$ PLAL after 30 min of irradiation. (d) HRTEM of CNp indicating lattice parameters.

Therefore, the effect of CNp functionalized laser textured substrates was investigated by culturing HaCaT cells on these test surfaces for 7 days. In this study, HaCaT cells were used as the biological model. This cell line is a well-described model to study the topographical influence on cell morphology and the organization of the cytoskeleton. Impact on surface topography was characterized in terms of the cell density and viability after 7 days incubation. The cell nuclei were stained with NucBlue Live ReadyProbes Reagent and florescence microscopy was performed to visualize the cell density and nuclear morphology (Fig. 8). Denser population of cells can be seen inside the channel compared to non-textured area between the channels. This preferential growth of the cells on the textured microchannels can be attributed to the combined effect of induced oxygen groups on COP due to laser processing as discussed in Section 3.1., increased roughness and surface area. ${ }^{33}$

The viability of HaCaT cells grown for 7 days on our patterned surface which was functionalized with laser generated NPs was investigated by a live/dead viability assay. The live/ dead viability assay showed that most of the cells were viable on the patterned NP functionalized surface (green) (Fig. 9a) with few circled dead cells represented in red (Fig. 9b).

The live/dead viability assays revealed healthy proliferation of cells well spread on the test substrate with high proportion of live cells. It should be noted that a high density of live cells was observed primarily in the textured microchannels. Additionally, good cell adhesion and cytoplasmic extension of keratinocyte cells can be seen (Fig. 9a). The high cell numbers with normal nuclear morphology and high viability after 7 days incubation suggest that our low cost and simple approach can be utilized to fabricate substrates for studying complex cellular interactions or cell responses without the use of any complicated multi-step lithographic processes. ${ }^{34}$

Following the cell viability and proliferation studies, further measurements were performed on the test surfaces to study the effect of different concentration of PLAL CNps on HaCaT cells (Fig. 10). The CNps were obtained for this study were produced by same technique as described in Section 3.3. It should be
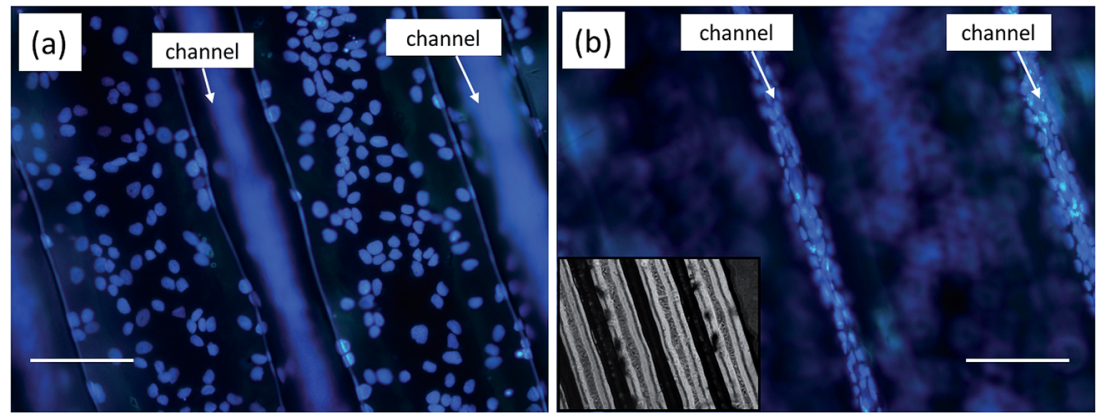

Fig. 8 Fluorescence microscopic images of HaCaT cells stained nuclei (blue) on NP functionalized laser textured substrates (a) focus on interchannel (b) focus on the channel. Inset (b) phase microscopy image of the channels, scale bar is $100 \mu \mathrm{m}$. 


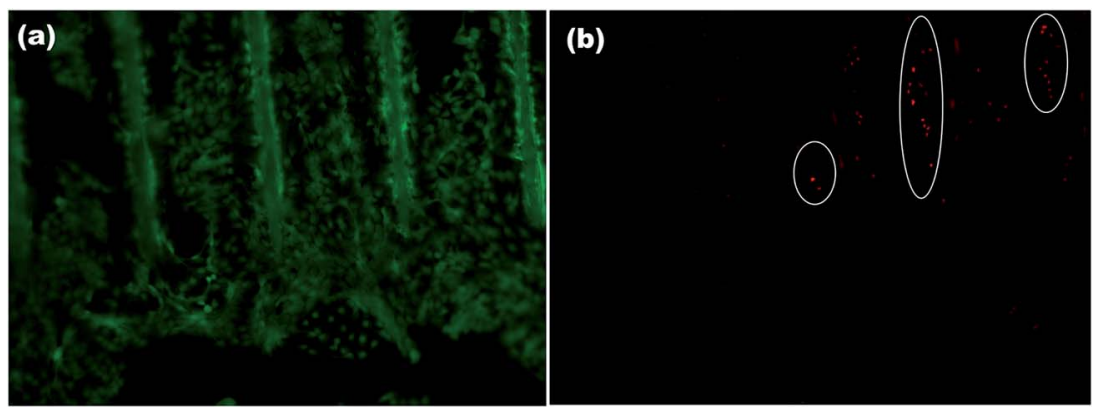

Fig. 9 LIVE/DEAD fluorescent viability assay after 8 days indicating (a) the presence of live cells in green and (b) dead cells represented in red highlighted within circle.
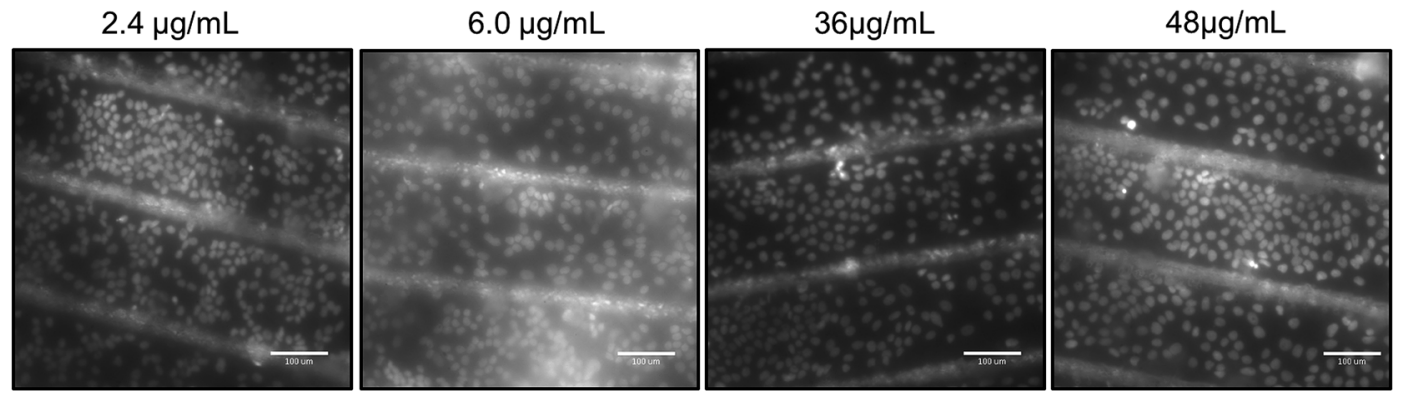

Fig. 10 Fluorescent microscopy images of nuclei stained $\mathrm{HaCaT}$ cells after exposure to different concentration of $\mathrm{CNps}$. The length of the scale bars in the micrograph images is $100 \mu \mathrm{m}$.

noted that to achieve comparative final surface composition, the amount of nanoparticle solution of different concentrations deposited on each surfaces' was kept constant i.e. $1 \mathrm{~mL} / 1 \mathrm{~cm}^{2}$.

Not many studies have evaluated concentration dependent toxicity effect of CNps on HaCaT cell line; most of the reported findings are confined to multi or single wall carbon nanotubes (CNTs) ${ }^{35,36}$ Our examination revealed that after 7 days exposure of HaCaT cells to $2.4,6.0,12,36,48 \mu \mathrm{g} \mathrm{mL}{ }^{-1} \mathrm{CNps}$, no significant difference in the cell growth was observed (refer Table $2 \mathrm{~S}$ in ESI $\dagger$ ). The shape of the cell nuclei were round and appeared healthy even for the highest dose $\left(48 \mu \mathrm{g} \mathrm{mL}{ }^{-1}\right)$ of CNps. In control experiments performed using non-textured nonfunctionalized COP substrates, cells did not appear to attach and proliferate compared to the NP functionalized and textured surfaces (Fig. 8S in ESI $\dagger$ ).

Taken together, these results validate that our test surfaces facilitate proliferation of HaCaT keratinocytes and induce the preferential growth in the laser textures microchannels. Therefore, these surfaces can be a promising candidate to investigate and mimic cells responses on biocompatible surfaces providing favorable physical/chemical characteristics.

Additionally, the properties of our platform can be tuned by functionalizing with different nanoparticle material to impart desired properties, thus presenting a versatile biocompatible platform. For proof of concept, titanium oxide (TiOx) and silicon oxide (SiOx) nanoparticles were chosen as a result of their successful demonstration in advanced applications such as bio-sensing, diagnostic and therapeutic applications. TiOx
NPs and SiOx NPs were prepared with same technique as for CNps discussed previously i.e. PLAL in DI-water by ablating targets of titanium and silicon respectively. Subsequently, similar set of biocompatibility studies were performed on TiOx NPs and SiOx NPs functionalized substrates as for CNps discussed in the above section. Similar results were observed, and no evident cytotoxicity was recorded (Fig. 9S in ESI $\dagger$ ).

\section{Conclusions}

A simple, innovative and low cost biomimetic platform based on laser processing and nanoparticle functionalization has been developed. Clean, uniform microchannels with tunable depth and width were successfully generated on an optically transparent polymer with an industrial $1064 \mathrm{~nm}$ Nd:YAG laser. Substrate surface chemistry was revealed by water contact angle measurements, XPS and NMR spectroscopies. Ultrapure and ligand free carbon nanoparticles were produced by pulsed laser ablation in DI water and were deposited onto laser textured surface to create a functional bio-platform. Cell attachment, viability and live/dead assay with HaCaT cell line demonstrated the biocompatibility of the developed substrate. The results on effect of CNps dose on cultured HaCaT keratinocytes revealed that particles concentration up to $48 \mu \mathrm{g} \mathrm{mL}^{-1}$ did not induce any toxic effects or cell damage. These results open up new challenging future work on examination of a wide range of potential applications of these platforms for biosensing and diagnostics. With the current technological advances, this 
innovative laser processing technology in future when combined with microfluidic device or thin film chromatographic systems may present novel design of optical biosensors for detection/separating pathogens, drugs and other chemical or biological species.

\section{Acknowledgements}

Authors would like to acknowledge Mr Cian Hughes for NMR spectroscopy experiments. This publication has emanated from research conducted with the financial support of the European Union's Horizon 2020 Research and Innovation Programme under the Marie Sklodowska-Curie grant agreement No. 655194 and Science Foundation Ireland (SFI) under Grant Number 12/IA/1576.

\section{References}

1 R. ltd, http://www.Researchandmarkets.com, 2017.

2 N. G. Durmuş, R. L. Lin, M. Kozberg, D. Dermici, A. Khademhosseini and U. Demirciet, Acoustic-Based Biosensors, in Encyclopedia of Microfluidics and Nanofluidics, ed. D. Li, Springer Science + Business media, New York, 2015, pp. 28-40.

3 C. Shan, H. Yang, D. Han, Q. Zhang, A. Ivaska and L. Niu, Langmuir, 2009, 25, 12030-12033.

4 R. Riahi, P. Gogoi, S. Sepehri, Y. Zhou, K. Handique, J. Godsey and Y. Wang, Int. J. Oncol., 2014, 1870-1878.

5 T. Nielsen, D. Nilsson, F. Bundgaard, P. Shi, P. Szabo, O. Geschke and A. Kristensen, J. Vac. Sci. Technol., B: Nanotechnol. Microelectron.: Mater., Process., Meas., Phenom., 2004, 22, 1770.

6 V. R. Sastri, in Plastics in Medical Devices Properties, Requirements and Applications, ed. W. Andrew, Applied Science Publishers, United Kingdom, 2nd edn, 2014, pp. 73-120.

7 A. Azouz, S. Murphy, S. Karazi, M. Vázquez and D. Brabazon, Mater. Manuf. Processes, 2014, 29, 93-99.

8 S. Prakash, B. Acherjee, A. Kuar and S. Mitra, Proc. Inst. Mech. Eng., Part B, 2013, 227, 508-519.

9 O. Lyutakov, J. Tůma, I. Huttel, V. Prajzler, J. Siegel and V. Švorčík, Appl. Phys. B, 2013, 110, 539-549.

10 P. Vikesland and K. Wigginton, Environ. Sci. Technol., 2010, 44, 3656-3669.

11 N. Tansil and Z. Gao, Mater. Today, 2006, 1, 28-37.

12 R. Intartaglia, A. Barchanski, K. Bagga, A. Genovese, G. Das, P. Wagener, E. Di Fabrizio, A. Diaspro, F. Brandi and S. Barcikowski, Nanoscale, 2012, 4, 1271.

13 V. Amendola, S. Scaramuzza, S. Agnoli, G. Granozzi, M. Meneghetti, G. Campo, V. Bonanni, F. Pineider,
C. Sangregorio, P. Ghigna, S. Polizzi, P. Riello, S. Fiameni and L. Nodari, Nano Res., 2015, 8, 4007-4023.

14 H. Zeng, X. Du, S. Singh, S. Kulinich, S. Yang, J. He and W. Cai, Adv. Funct. Mater., 2012, 22, 1333-1353.

15 S. Ibrahimkutty, P. Wagener, T. Rolo, D. Karpov, A. Menzel, T. Baumbach, S. Barcikowski and A. Plech, Sci. Rep., 2015, 5, 16313.

16 P. Innocenzi, L. Malfatti and D. Carboni, Nanoscale, 2015, 7, 12759-12772.

17 Y. Sun, X. Wang, F. Lu, L. Cao, M. Meziani, P. Luo, L. Gu and L. Veca, J. Phys. Chem. C, 2008, 112, 18295-18298.

18 P. van Midwoud, A. Janse, M. Merema, G. Groothuis and E. Verpoorte, Anal. Chem., 2012, 84, 3938-3944.

19 P. Nunes, P. Ohlsson, O. Ordeig and J. Kutter, Microfluid. Nanofluid., 2010, 9, 145-161.

20 T. Stachowiak, D. Mair, T. Holden, L. Lee, F. Svec and J. Fréchet, J. Sep. Sci., 2007, 30, 1088-1093.

21 S. Gibbs, Skin Pharmacol. Physiol., 2009, 22, 103-113.

22 P. Boukamp, J. Cell Biol., 1988, 106, 761-771.

23 O. Loebich, Gold Bull., 1972, 5, 2-10.

24 R. McCann, K. Bagga, R. Groarke, A. Stalcup, M. Vázquez and D. Brabazon, Appl. Surf. Sci., 2016, 387, 603-608.

25 R. Suriano, A. Kuznetsov, S. Eaton, R. Kiyan, G. Cerullo, R. Osellame, B. Chichkov, M. Levi and S. Turri, Appl. Surf. Sci., 2011, 257, 6243-6250.

26 T. Barr and S. Seal, J. Vac. Sci. Technol., A, 1995, 13, 12391246.

27 B. Wang, X. Wang, H. Zheng and Y. Lam, Nanomaterials, 2015, 5, 1442-1453.

28 D. Eddington, J. Puccinelli and D. Beebe, Sens. Actuators, B, 2006, 114, 170-172.

29 K. Bagga, R. McCann, M. Wang, A. Stalcup, M. Vázquez and D. Brabazon, J. Colloid Interface Sci., 2015, 447, 263-268.

30 D. Wu, X. Huang, X. Deng, K. Wang and Q. Liu, Anal. Methods, 2013, 5, 3023.

31 J. Wang, C. Wang and S. Chen, Angew. Chem., Int. Ed., 2012, 124, 9431-9435.

32 H. Li, Z. Kang, Y. Liu and S. Lee, J. Mater. Chem., 2012, 22, 24230 .

33 J. Jeon, S. Chung, R. Kamm and J. Charest, Biomed. Microdevices, 2010, 13, 325-333.

34 J. Stroble, R. Stone and S. Watkins, Sens. Rev., 2009, 29, 112119.

35 X. Hu, S. Cook, P. Wang, H. Hwang, X. Liu and Q. Williams, Sci. Total Environ., 2010, 408, 1812-1817.

36 E. Herzog, A. Casey, F. Lyng, G. Chambers, H. Byrne and M. Davoren, Toxicol. Lett., 2007, 174, 49-60. 